\section{Step by step}

"Well, it's in press, but if you want to try the technique we've developed, I'd rather send you my lab notebook version; there are details in there that will make a difference." This statement, overheard recently at a poster session, is symptomatic of the problems faced by scientists trying to master constantly evolving techniques. Methods sections of traditional papers, while providing the minimal information necessary to understand how experiments were conducted, are simply not adapted to convey the technical subtleties necessary to reproduce an unfamiliar procedure. But help is on the way.... As this journal goes to press, Nature Publishing Group is adding the finishing touches before the June launch of a new initiative, distinct from but complementary to Nature Methods, taking advantage of the online format to facilitate sharing of laboratory procedures.

Because of its emphasis, Nature Methods has always published extensive details on the novel methods described in its Articles, and sometimes asks authors to provide step-by-step procedures in supplementary information. In addition, the journal features a Protocols section describing established methods in 'bench terms'. Expanding on this concept, the new online resource has two main components: Nature Protocols and Protocols Network. The first component will feature a rapidly growing collection of authoritative Protocols similar to those found in Nature Methods, in an even broader range of topics and including both recently developed procedures and 'old classics'. The online format lends itself well to interactive features such as keyword search and posting of comments.

Nature Methods will continue to publish Protocols, usually one per issue, focusing on recent techniques of particularly broad interest or especially timely for a subset of our audience. Although Nature Methods and Nature Protocols are editorially independent, the Protocols published in Nature Methods will soon be searchable via Nature Protocols. In the near future, a keyword search for protocols on the new website will highlight relevant search results from both titles.

The second component of the new website, Protocols Network, is designed as a community platform allowing everyone to easily upload their own protocols and share them with their peers. In contrast to Nature Protocols, which requires an institutional subscription, Protocols Network will be freely accessible. The authors of papers in all Nature journals are now encouraged to make available the procedures used in their articles by uploading them to the Protocols Network. With electronic links between the procedures in the Protocols Network and the corresponding paper in the journal, this is a solution to the dilemma between succinctness of a paper and its methodological richness. Moreover, users of the site will be able to post comments on Protocols, thus creating an interactive environment conducive to exchange of ideas, efficient troubleshooting and ultimately improvement of methods.

Nature Protocols is not the only program to present laboratory procedures online (Nature 441, 678; 2006). For several years, online versions of Current Protocols manuals have been available. And at the beginning of June, Cold Spring Harbor Laboratory Press launched CSH Protocols, offering electronic versions of some of their laboratory manuals with plans to complement these with new, original protocols.

A growing number of community-specific initiatives also make protocols available on the web. For example, the Alliance for Cell Signaling posts protocols in an effort to standardize the way their members conduct collaborative studies. On a smaller scale, 'The Epigenome', one of the 'Networks of Excellence' funded by the European Community, posts selected and tested protocols as a trusted reference for their community.

Although one might regret the lack of centralization, the development of more initiatives for the dissemination of quality laboratory procedures is good news for scientists. And as with cookbooks, the interest may also lie in comparing several trusted sources. To celebrate the occasion, we have boosted the Protocols section this month and present three Protocols on various applications of a technology ripe for propagation: emulsion technology or, as the authors of the accompanying Overview put it, the science of 'mayonnaise' (p. 541). This technique, already at the core of the new generation of DNA sequencers, is also perfectly adapted to the challenges of directed evolution of enzymes (p. 561) and quantification of rare genetic variants (p. 551).

In addition to these sophisticated pursuits, emulsion technology can also improve ubiquitous laboratory applications such as the amplification of DNA libraries (p. 545). But making the mayonnaise can be an intimidating endeavor for the noninitiated cook, and we hope that these Protocols will help disseminate the technique for the benefit of many researchers.

So please visit Nature Protocols (http://www. natureprotocols.com), contribute to Protocols Network and indulge yourself with a dollop of mayonnaise on your PCR! 